\title{
On the Probabilistic Epipolar Geometry
}

\author{
Sami S. Brandt \\ Helsinki University of Technology \\ Laboratory of Computational Engineering \\ P.O. Box 9203, FIN 02015 HUT, FINLAND \\ Sami.Brandt@hut.fi
}

\begin{abstract}
In this paper, we are going to answer the following question: assuming that we have estimates for the epipolar geometry and its uncertainty between two views, how probable it is that a new, independent point pair will satisfy the true epipolar geometry and be, in this sense, a feasible candidate correspondence pair? If we knew the true fundamental matrix, the answer would be trivial but in reality it is not because of estimation errors. So, as a point in the first view is given, we will show that we may compute a probability density for the feasible correspondence locations in the second view that describes the current level of knowledge of the epipolar geometry between the views. We will thus have a point-probability-density relation which can be understood as a probabilistic form of the epipolar constraint; it also approaches the true point-line relation as the number of training correspondences tends to infinity. We will also show that the eigenvectors of the epipolar line covariance matrix have certain interpretations on the image plane, of which one is the previously observed, narrowest point of the epipolar envelope. The results of this paper are novel and important since the uncertainty of the epipolar constraint can be now taken into account in a sound way in applications.
\end{abstract}

\section{Introduction}

Epipolar geometry helps in matching points between two views since the correspondence for a point in the first view must lie on the corresponding epipolar line in the second view. However, when the epipolar geometry is estimated from noisy data, the true, noise free matches will not lie on the estimated epipolar lines precisely, since the fundamental matrix will be known only up to a finite level of accuracy. Typically, the deviation from the estimated epipolar lines is especially large when a true correspondence pair is clearly out of the disparity range of the training correspondences. One key question posed in this paper is thus what can we say about the probability of a point pair to satisfy the true epipolar geometry given estimates of the epipolar geometry and its uncertainty that are computed from some independent sample correspondences between the views.

The starting point for this work was the results in $[12,13,5]$, where it was noticed that the error bounds of the epipolar lines seem to be narrowest at the most probable location of the match. They suggested that the covariance matrix $[4,7]$ of the fundamental matrix might therefore capture disparity information of the training scene, although the epipolar geometry itself reveals nothing about the disparity. However, an accurate explanation for the narrowest point of the envelope has not been given so far [6]. Consequently in Section 2, we will first show formally that these conjectures are right and show how this 
most probable location can be computed. The most probable point should be seen as the point which will most probably, given the so far gatherer evidence of the epipolar geometry, be on the true epipolar line-not as a prediction of disparity when we extend the discussion to a new scene with different depth distribution from the training scene.

In total, this paper considers the use of covariance matrix of the fundamental matrix by studying the epipolar line variation in the dual space but similar analysis can be performed for uncertain lines in general. In Section 3, we transform the epipolar line variation, which is a dual, line distribution, to a point distribution onto the image plane. This is an exact probabilistic version of the epipolar constraint: it is represented by the pointprobability-density relation and, as our information of the epipolar geometry grows, in general, the point probability-density will converge to the true epipolar line as an improper point density. The derived probability density will just tell that the true epipolar line points, corresponding to a point in the first view, can be anywhere in the second image as our knowledge of the epipolar geometry is uncertain, but, points on certain finite regions in the second image satisfy the true epipolar geometry more probably than on some others.

Where previous applications frequently assume that the estimated epipolar geometry is correct and neglect the fact that even the maximum likelihood estimates for the epipolar lines deviate from the true epipolar lines, this paper places in between unknown and certain epipolar geometry. The motivation is in reality where estimated epipolar geometry is never completely error free, hence a single epipolar line estimate does not tell the whole truth of the revealed epipolar geometry. In matching, for instance, extrapolation of the epipolar constraint outside the disparity range of the training correspondences is evidently risky, but previously there has been no clear means to measure this risk. If we replace the epipolar line estimates by the derived point probability-densities, we may use uncertain epipolar geometry in a sound way in applications by using the probability theory as the logic. Moreover, will not say more of the constraint than there has been evidence of it in the training data. Nevertheless, we do not claim that the derived probability density would be the only available cue, say, in matching. Other priors, e.g. for disparity, can and should be additionally used while all the cues can be exploited by the probability theory.

Recently, Triggs proposed a method that models the joint probability distributions of correspondences [10] that are aimed at summarising the behaviour of the training correspondences but not to rigidly constrain them to an ideal predefined geometry. In both, his and our approaches, a loose analogy can be seen to Bayesian inference in the form of model averaging [9] and marginalisation. The difference between our and Triggs' approach [10] is significant since he just summarises the feature distributions by Gaussians on the image planes, and considers an algebraic linear system for their estimation. We, however, derive our distributions in the dual space from the epipolar line covariance and the related Gaussian model which is justified by the general estimation theory since maximum likelihood parameter estimates are asymptotically normally distributed. With our approach, we may additionally use any statistically sound criterion for the fundamental matrix and its covariance, and are able to estimate them robustly [3, 1, 2].

\section{Special Points and Lines}

In this section, we will show that certain points and lines in the image have a special role as soon as the epipolar line variation approaches to a normal distribution in the dual space. To create a convenient parameterisation for lines, we follow [6] by defining the point-line 
relation of the epipolar geometry by the mapping $\mathbf{l}: \mathbb{P}^{2} \longrightarrow \mathbb{R}^{3}$ such that $\mathbf{l}(\mathbf{x}) \triangleq\|\mathbf{F x}\|^{-1} \mathbf{F x}$. The first-order covariance approximation for the epipolar line $\mathbf{l}$ in the second image, given the point $\mathbf{x}$ in the first, is

$$
\mathbf{C}_{\mathbf{l}} \simeq \frac{\partial \mathbf{l}}{\partial \mathbf{F}} \mathbf{C}_{\mathbf{F}} \frac{\partial \mathbf{l}}{\partial \mathbf{F}}^{\mathrm{T}}+\frac{\partial \mathbf{l}}{\partial \mathbf{x}} \mathbf{C}_{\mathbf{x}} \frac{\partial \mathbf{l}}{\partial \mathbf{x}}^{\mathrm{T}},
$$

where $\partial \mathbf{l} / \partial \mathbf{F}$ is the Jacobian of the point-line mapping above with respect to the elements of $\mathbf{F}$, and $\mathbf{x}$ is assumed to be contaminated by Gaussian noise with covariance matrix $\mathbf{C}_{\mathbf{x}}$.

Proposition 1 Let $\mathbf{x}$ be a point in the first view, corrupted by Gaussian noise with covariance matrix $\mathbf{C}_{\mathbf{x}}$; let $\hat{\mathbf{F}}$ be the estimated fundamental matrix with estimated covariance matrix $\hat{\mathbf{C}}_{\mathbf{F}}$, where the estimation is performed independently of $\mathbf{x}$. Then, up to the first-order, Gaussian approximation, the eigenvectors, sorted in descending order of the eigenvalues, of the epipolar line covariance matrix $\hat{\mathbf{C}}_{\mathbf{l}}$, corresponding to $\mathbf{x}$ and having the rank of 2 with no multiple eigenvalues, have the following interpretations in the second image:

$$
\begin{gathered}
\mathbf{u}_{1} \text { : the least probable pencil of epipolar lines containing } \mathbf{l}_{0} \text {; } \\
\text { the least probable epipolar line intersecting the point } \mathbf{u}_{2} \\
\mathbf{u}_{2} \text { : the most probable pencil of epipolar lines; } \\
\text { the least probable epipolar line intersecting the point } \mathbf{u}_{1} \\
\mathbf{u}_{3} \text { : the least probable pencil of epipolar lines; } \\
\text { the mean epipolar line estimate } \mathbf{l}_{0} \text { corresponding to } \mathbf{x} .
\end{gathered}
$$

Proof. Since the first-order approximation for $\mathbf{C}_{\mathbf{1}}$ is equivalent to its tangential approximation at the estimated (mean) epipolar line $\mathbf{l}_{0}=\|\mathbf{F x}\|^{-1} \mathbf{F x}$, the covariance matrix approximation is degenerate and positive semi-definite. Since it is also real and symmetrical, we may perform the similarity transform and define

$$
\mathbf{C}_{\mathbf{l}}^{\prime}=\mathbf{U}^{\mathrm{T}} \mathbf{C}_{\mathbf{l}} \mathbf{U}=\left(\begin{array}{ccc}
\sigma_{1}^{2} & 0 & 0 \\
0 & \sigma_{2}^{2} & 0 \\
0 & 0 & 0
\end{array}\right)
$$

where $\mathbf{U}$ contains the eigenvectors of $\mathbf{C}_{\mathbf{1}}$ normalised to the unit norm and ordered such that $\sigma_{1} \geq \sigma_{2}$. We notice that the mean epipolar line $\mathbf{l}_{0}$ belongs to the left null space of the Jacobians $\partial \mathbf{l} / \partial \mathbf{F}$ and $\partial \mathbf{l} / \partial \mathbf{x}$; so it must also belong to the null space of $\mathbf{C}_{\mathbf{l}}$, hence, $\mathbf{u}_{3} \equiv \mathbf{l}_{0}$. Let the tangent plane of the unit sphere at $\mathbf{u}_{3}$ be $\pi$ and the tangent plane at $\mathbf{e}_{3}=\left(\begin{array}{lll}0 & 0 & 1\end{array}\right)^{\mathrm{T}}$ be $\pi^{\prime}$. If we regard $\mathbb{R}^{3}$ as a projective space $\mathbb{P}^{2}, \mathbf{U}^{\mathrm{T}}$ can be also seen as the collineation $\mathbf{U}^{\mathrm{T}}: \mathbb{P}^{2} \longrightarrow \mathbb{P}^{2}$ from the projective plane $\pi$ to $\pi^{\prime}$, i.e., the lines (and points) transform according to $\mathbf{l}^{\prime}=\mathbf{U}^{\mathrm{T}} \mathbf{l}$. The mean epipolar line is the origin in the transformed dual space $\pi^{\prime}$ since $\mathbf{e}_{3}=\mathbf{U}^{\mathrm{T}} \mathbf{u}_{3}$. Correspondingly, the direction of the largest variance $\mathbf{u}_{1}$ is transformed to $\mathbf{e}_{1}=\left(\begin{array}{lll}1 & 0 & 0\end{array}\right)^{\mathrm{T}}$ and the direction of the smaller variance $\mathbf{u}_{2}$ to $\mathbf{e}_{2}=\left(\begin{array}{lll}0 & 1 & 0\end{array}\right)^{\mathrm{T}}$.

It is assumed that the covariance is non-isotropic in the sense that $\sigma_{1}>\sigma_{2}$. Therefore, in the transformed dual space the most probable line is the $x$-axis $\left(\begin{array}{lll}0 & 1 & 0\end{array}\right)^{\mathrm{T}} \equiv \mathbf{e}_{2}$, since the marginal probability or integral of the two-dimensional (degenerate three-dimensional) Gaussian distribution with the covariance matrix $\mathbf{C}_{1}^{\prime}$ centred at $\mathbf{e}_{3}$ is the largest over this line (see Fig. 1). The most probable point in the image hence is $\mathbf{u}_{2}=\mathbf{U} \mathbf{e}_{2}$ in the sense that the total probability of the pencil of epipolar lines intersecting $\mathbf{u}_{2}$ is the largest. Since the (dual) line $\mathbf{e}_{2}$ intersects the origin, it contains the (dual) point of the mean epipolar line, and the point $\mathbf{u}_{2}$ is therefore also on the estimated epipolar line. The least probable 


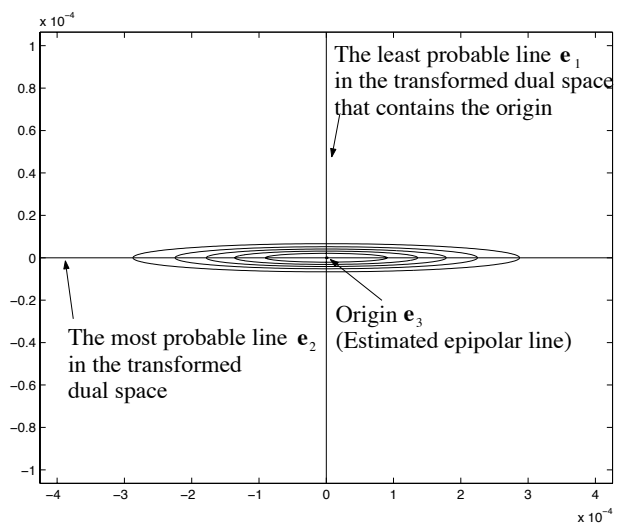

Figure 1: Illustration of the transformed dual space or the projective plane $\pi^{\prime}$ with the contours of the Gaussian corresponding to $\mathbf{C}_{\mathbf{l}}^{\prime}$. The estimated epipolar line $\mathbf{l}_{0}$ corresponds to the origin $\mathbf{e}_{3}$, and the most probable point in the image space is $\mathbf{u}_{2}=\mathbf{U} \mathbf{e}_{2}$ since the marginal probability over the line $\mathbf{e}_{2}$ is the largest. Correspondingly, the least probable point on the estimated epipolar line is $\mathbf{u}_{1}=\mathbf{U} \mathbf{e}_{1}$ since $\mathbf{e}_{1}$ has the smallest total probability of the lines that contain the origin.

point on the estimated epipolar line is correspondingly the $y$-axis or the line $\mathbf{e}_{1}$ in the transformed dual space since the marginal probability is the smallest of all (dual) lines that intersect the origin $\mathbf{e}_{3}$. Hence, the least probable pencil of epipolar lines that contain the line $\mathbf{u}_{3}$ must be $\mathbf{u}_{1}=\mathbf{U} \mathbf{e}_{1}$. The least probable line in the transformed dual space is the line at infinity $\mathbf{e}_{3}$, its marginal probability being zero; hence the point interpretation of $\mathbf{u}_{3}$ is the least probable pencil of epipolar lines in the second image. In the transformed dual space, $\mathbf{e}_{1}$ is the least probable point on the $x$-axis because, as an ideal point it has zero probability density value. Its line interpretation $\mathbf{u}_{1}$ in the second image is hence the least probable line that intersects the most probable point $\mathbf{u}_{2}$. Correspondingly, $\mathbf{e}_{2}$ is the least probable point on the $y$-axis in the dual space, and therefore $\mathbf{u}_{2}$ is the least probable epipolar line for $\mathbf{x}$ that intersects the point $\mathbf{u}_{1}$.

A crucial assumption is that the non-zero eigenvalues of the epipolar line covariance matrix are not equal. If they were, no line intersecting the origin in the transformed dual space would be in a more probable position. In other words, every pencil of lines centred at any point on the mean epipolar line would share equal total probability. Nevertheless, the F-matrix is normally estimated from point matches with concentrated disparity distribution, which suggests that $\sigma_{1} \gg \sigma_{2}$, which can be easily verified experimentally.

In Fig. 2, we have an example of how the point and line interpretations of the eigenvectors of the epipolar line covariance are related. Since the distance between the origin and the estimated epipolar line is large, the point interpretation of $\mathbf{u}_{3}$ is very close to the origin. This example shows that, while the origin may be fixed to an arbitrary point in the image, its location exerts a special influence, and affects the eigenvectors of $\mathbf{C}_{\mathbf{1}}$ and hence the location of the special points and lines in the image, except $\mathbf{l}_{0}$, and even the epipolar envelopes, which is not desirable. Another way to see this is the known fact that error covariances are not invariant to coordinate transforms, thus covariances may look completely different in different frames [11] - this may also be seen as an opportunity since we may choose the coordinate system such that our density approximation, which can be also seen as the Laplace's method [8], is most accurate. In fact, a careful analytical and experimental analysis shows [2] that a most reasonable way of selecting the coordinate 


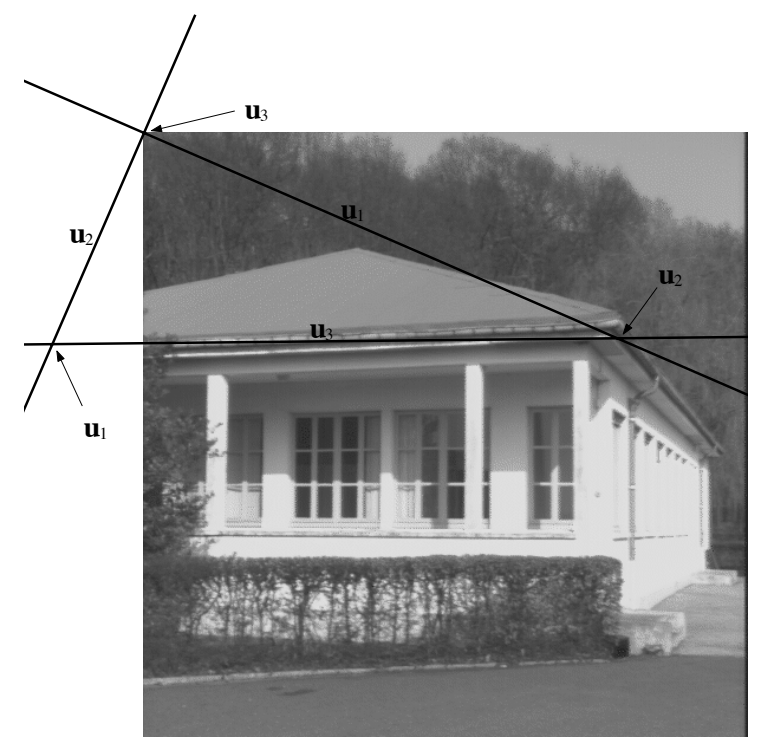

Figure 2: Point and line interpretations of the eigenvectors of the epipolar line covariance matrix $\mathbf{C}_{\mathbf{l}}$ that is computed for the corner point in the cornice of the roof. As expected, the most probable point $\mathbf{u}_{2}$ is close to the true correspondence point. Since the estimated epipolar line $\mathbf{l}_{0} \equiv \mathbf{u}_{3}$ is far away from the origin, the point interpretation of $\mathbf{u}_{3}$ is correspondingly very close to the origin. This makes the origin a special point of the image. INRIA Syntim owns the copyright of the stereo pair.

system is to simply translate the image origin far away from the mean epipolar line.

Let us now return to the previously made hypothesis that the match location is with higher probability at the narrowest point of the epipolar envelope since we are now able to explain this observation. The confidence intervals of the epipolar lines in the first-order approximation are represented by the conic [6]

$$
\mathbf{C}=\mathbf{l}_{0} \mathbf{l}_{0}^{\mathrm{T}}-k^{2} \mathbf{C}_{\mathbf{l}}
$$

where $k^{2}$ follows the cumulative $\chi_{n}^{2}$ distribution with two degrees of freedom. We observe that the line interpretation of $\mathbf{u}_{2}$ is polar of the point $\mathbf{u}_{2}$ with respect to the conic $\mathbf{C}$ (see Fig. 3) because $\mathbf{C}$ has identical eigenvectors to $\mathbf{C}_{\mathbf{1}}$; hence, $\mathbf{u}_{2} \hat{=} \mathbf{C} \mathbf{u}_{2}$ (equality up to scale). Let us additionally assume that the epipolar envelope is a hyperbola of two sheets, as is normally the case. Since the least probable epipolar line is normally far away from the most probable point, the tangency lines from the most probable point are close to asymptotes of the hyperbola. The centre of the hyperbola is the intersection of its asymptotes, i.e., the line at infinity is polar to the centre. The centre of the hyperbola is therefore close to the most probable point, but they do not coincide unless the line $\mathbf{u}_{2}$ is the line at infinity.

\section{Derivation of the Probabilistic Epipolar Constraint}

In this section, we will further develop the considerations presented in Section 2 and derive the probabilistic epipolar constraint. The fundamental observation is that the probability of any point $\mathbf{x}^{\prime}$ in the second image, to correspond to the point $\mathbf{x}$ in the first view, is equivalent to the total probability of those epipolar geometries that would explain the correspondence $\mathbf{x} \leftrightarrow \mathbf{x}^{\prime}$ in the two views. In other words, we compute the total probability of the pencil of (epipolar) lines centred at $\mathbf{x}^{\prime}$, or the total probability of the line $\mathbf{x}^{\prime}$ in the 


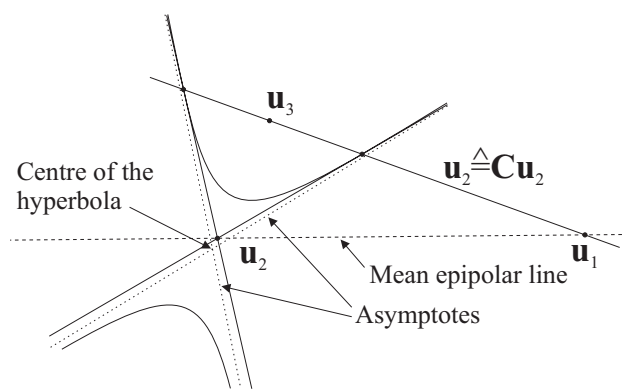

Figure 3: By the pole-polar relationship, any point $\mathbf{x}$ outside the conic $\mathbf{C}$ induces a polar line $\mathbf{l}=\mathbf{C x}$ that intersects the conic at two points, and the tangents at these points intersect at $\mathbf{x}$. Since here $\mathbf{u}_{2}$ is an eigenvector of the epipolar envelope $\mathbf{C}$ and is outside the conic, its line interpretation is polar to its point interpretation. Moreover, if $\mathbf{C}$ is a hyperbola and the point $\mathbf{u}_{3}$ is far away from the epipolar line the tangent lines of $\mathbf{C}$ at the intersection points of the line $\mathbf{u}_{2}$ and $\mathbf{C}$ are close to the asymptotes of $\mathbf{C}$. The centre of the hyperbola, the intersection of those tangent lines whose points of tangency are on $\mathbf{l}_{\infty}$, is then close to the point $\mathbf{u}_{2}$.

dual space that is, in principal, the marginal probability of the Gaussian model over the line $\mathbf{x}^{\prime}$ in the dual space. The corresponding transform of the dual probability density is, in fact, similar to the well-known Radon transform, but lines must be weighted on the basis of their direction here.

Proposition 2 Let $\mathbf{x}$ be a point in the first view and assume that the epipolar geometry and its covariance matrix has been estimated independently of $\mathbf{x}$. Let the epipolar line covariance matrix corresponding to $\mathbf{x}$ be $\mathbf{C}_{1}$, defined as in (1) and (2). Then, up to a Gaussian approximation, $\mathbf{C}_{\mathbf{1}}$ determines a $2 \mathrm{D}$ probability density for the epipolar line points $\mathbf{x}^{\prime}$ in the second view and the density is represented by

$$
p\left(r, \theta \mid \mathbf{C}_{\mathbf{l}}\right)=\frac{\sigma_{1} \sigma_{2} \mathrm{e}^{-\frac{1}{2} r^{-2}\left(\sigma_{1}^{2} \cos ^{2} \theta+\sigma_{2}^{2} \sin ^{2} \theta\right)^{-1}}}{\sqrt{2 \pi^{3}} r^{2}\left(\sigma_{1}^{2} \cos ^{2} \theta+\sigma_{2}^{2} \sin ^{2} \theta\right)^{3 / 2}},
$$

where the point $\mathbf{x}^{\prime}$ is parameterised with the signed-distance-direction pair $(r, \theta)$ on the transformed image plane $\pi^{\prime}$.

Proof. In this proof, we use several times the property that when we have an almost everywhere continuous and invertible mapping $\mathbf{s}: S \longrightarrow R$ in any set $S \subset \mathbb{R}^{n}$, we may evaluate the integral of the kernel $p(\mathbf{s})$ by substitution $\mathbf{s}=\mathbf{s}(\mathbf{r})$ in the domain $R$ and it holds $\int_{S} p(\mathbf{s}) \mathrm{d} \mathbf{s}=\int_{R} p(\mathbf{s}(\mathbf{r}))|\operatorname{det} \mathbf{J} \mathbf{s}(\mathbf{r})| \mathrm{d} \mathbf{r}$, where $\mathrm{J}$ is the Jacobian operator.

Let us transform the original coordinate system by the orthogonal transform $\mathbf{U}^{\mathrm{T}}$, where $\mathbf{U}$ contains the unit eigenvectors of $\mathbf{C}_{\mathbf{l}}$ in the descending order of the eigenvalues; hence $\mathbf{x}^{\prime \prime} \triangleq \mathbf{U}^{\mathrm{T}} \mathbf{x}^{\prime}, \mathbf{e}_{3}=\mathbf{U}^{\mathrm{T}} \mathbf{l}_{0}$, and $\mathbf{C}_{\mathbf{l}}^{\prime}=\mathbf{U}^{\mathrm{T}} \mathbf{C}_{\mathbf{l}} \mathbf{U}$ as in (2). Let us now consider the dual space of this transformed space. Let us write those lines of the transformed dual space that are parallel to $\mathbf{x}^{\prime \prime}$ in parametric form

$$
\mathbf{l}_{s}(t)^{\prime}=\left(\begin{array}{c}
t \mathbf{d}+s \mathbf{n} \\
1
\end{array}\right), \quad s, t \in \mathbb{R}
$$

where $\mathbf{d}$ is the unit direction vector of the line $\mathbf{x}^{\prime \prime}$ and $\mathbf{n}$ is its unit normal such that the polar angle of $\mathbf{d}$ is on $\left[-\frac{\pi}{2}, \frac{\pi}{2}\right)$ and $\operatorname{det}(\mathbf{d} \mathbf{n})=1$.

By assumption, the epipolar lines, corresponding to the point $\mathbf{x}$ in the first view, are normally distributed in the transformed dual space with the mean at $\mathbf{e}_{3}$ and covariance 
matrix $\mathbf{C}_{\mathbf{l}}^{\prime}$, and the corresponding density function is $p\left(\mathbf{l}_{s}(t)^{\prime} \mid \mathbf{e}_{3}, \mathbf{C}_{\mathbf{l}}^{\prime}\right)$. The marginal probability of $p\left(\mathbf{l}_{s}(t)^{\prime} \mid \mathbf{e}_{3}, \mathbf{C}_{\mathbf{l}}^{\prime}\right)$ over the lines parallel to $\mathbf{x}^{\prime \prime}$ depends only on the direction angle $-\theta^{\mathrm{c}} \in\left[-\frac{\pi}{2}, \frac{\pi}{2}\right)$ of the line $\mathbf{x}^{\prime \prime}$ where $\theta^{\mathrm{c}}=\frac{\pi}{2} \operatorname{sign} \theta-\theta$ is the complement angle of the direction $\theta$ of the point $\mathbf{x}^{\prime \prime}$, as we define sign $0=1$. The marginal probability is

$$
p\left(s \mid \theta, \mathbf{C}_{\mathbf{l}}^{\prime}\right) \triangleq \int_{-\infty}^{\infty} p\left(\mathbf{l}_{s}(t)^{\prime} \mid \mathbf{e}_{3}, \mathbf{C}_{\mathbf{l}}^{\prime}\right) \mathrm{d} t \equiv \int_{L} p\left(\mathbf{l} \mid \mathbf{l}_{0}, \mathbf{C}_{\mathbf{l}}\right) \mathrm{d} L
$$

Let us evaluate the kernel $p\left(\mathbf{l}_{s}(t)^{\prime} \mid \mathbf{e}_{3}, \mathbf{C}_{\mathbf{l}}^{\prime}\right)$ or the 2 D Gaussian $p\left(\tilde{\mathbf{l}}_{s}(t)^{\prime} \mid \mathbf{0}_{2}, \tilde{\mathbf{C}}_{\mathbf{l}}^{\prime}\right)$, where $\tilde{\mathbf{I}}_{s}(t)^{\prime}=$ $(t \mathbf{d}+s \mathbf{n}), \mathbf{0}_{2}=\left(\begin{array}{ll}0 & 0\end{array}\right)^{\mathrm{T}}$, and $\tilde{\mathbf{C}}_{\mathbf{l}}^{\prime}=\operatorname{diag}\left(\sigma_{1}^{2}, \sigma_{2}^{2}\right)$. Let us make the substitution

$$
\left(\begin{array}{l}
t \\
s
\end{array}\right)=\mathbf{R}^{\mathrm{T}} \tilde{\mathbf{C}}_{\mathbf{l}}^{\prime \frac{1}{2}} \mathbf{R}^{\prime}\left(\begin{array}{l}
u \\
v
\end{array}\right) \Leftrightarrow \tilde{\mathbf{I}}_{s}^{\prime}(t)=\tilde{\mathbf{C}}_{\mathbf{l}}^{\prime \frac{1}{2}} \mathbf{R}^{\prime}\left(\begin{array}{l}
u \\
v
\end{array}\right)
$$

where $\mathbf{R}=(\mathbf{d} \mathbf{n})$ and $\mathbf{R}^{\prime \mathrm{T}}=\left(\mathbf{d}^{\prime} \mathbf{n}^{\prime}\right)^{\mathrm{T}}$ is the rotation that brings the image of the line $\mathbf{x}^{\prime \prime}$ horizontal in the $u v$-plane, and the rotation angle is on the interval $\left[-\frac{\pi}{2}, \frac{\pi}{2}\right)$. Now,

$$
p\left(\tilde{\mathbf{l}}_{s}(t)^{\prime} \mid \mathbf{0}_{2}, \tilde{\mathbf{C}}_{\mathbf{l}}^{\prime}\right) \hat{=}\left|\operatorname{det}\left(\mathbf{R}^{\mathrm{T}} \tilde{\mathbf{C}}_{\mathbf{l}}^{\prime \frac{1}{2}} \mathbf{R}^{\prime}\right)\right| p\left(\tilde{\mathbf{C}}_{\mathbf{l}}^{\prime \frac{1}{2}} \mathbf{R}^{\prime}\left(\begin{array}{c}
u \\
v
\end{array}\right) \mid \mathbf{0}_{2}, \tilde{\mathbf{C}}_{\mathbf{l}}^{\prime}\right)=\frac{1}{2 \pi} \mathrm{e}^{-\frac{1}{2}\left(u^{2}+v^{2}\right)},
$$

where $\hat{=}$ denotes the equivalence between the two kernels. Hence,

$$
\int_{-\infty}^{\infty} p\left(\mathbf{l}_{s}(t)^{\prime} \mid \mathbf{e}_{3}, \mathbf{C}_{\mathbf{l}}^{\prime}\right) \mathrm{d} t \hat{=} \frac{1}{\sqrt{2 \pi}} \mathrm{e}^{-\frac{1}{2} v^{2}}
$$

Since $0=\mathbf{n}^{\mathrm{T}} \mathbf{d}=\mathbf{n}^{\mathrm{T}} \tilde{\mathbf{C}}_{\mathbf{l}}^{\prime \frac{1}{2}} \tilde{\mathbf{C}}_{\mathbf{l}}^{\prime-\frac{1}{2}} \mathbf{d}=\frac{\mathbf{n}^{\mathrm{T}} \tilde{\mathbf{C}}_{1}^{\prime \frac{1}{2}}}{\left\|\mathbf{n}^{\mathrm{T}} \tilde{\mathbf{C}}_{1}^{\prime \frac{1}{2}}\right\|} \frac{\tilde{\mathbf{C}}_{1}^{\prime-\frac{1}{2}} \mathbf{d}}{\left\|\tilde{\mathbf{C}}_{1}^{\prime-\frac{1}{2}} \mathbf{d}\right\|}=\mathbf{n}^{\prime \mathrm{T}} \mathbf{d}^{\prime}, \tilde{\mathbf{C}}_{\mathbf{l}}^{\prime-\frac{1}{2}}$ preserves the orientation, and $\mathbf{d}^{\prime}$ lies in the same quadrant as $\mathbf{d}$, we return to the original coordinate frame by the substitution $v=\mathbf{n}^{\prime \mathrm{T}} \tilde{\mathbf{C}}_{\mathbf{l}}^{\prime-\frac{1}{2}} \tilde{\mathbf{l}}_{s}^{\prime}(t)=s /\left\|\mathbf{n}^{\mathrm{T}} \tilde{\mathbf{C}}_{\mathbf{l}}^{\prime \frac{1}{2}}\right\|$. By denoting that $\mathbf{n}=\left(-\sin -\theta^{\mathrm{c}} \cos -\theta^{\mathrm{c}}\right)^{\mathrm{T}}=$ $\operatorname{sign} \theta(\cos \theta \sin \theta)^{\mathrm{T}}$ and $\left\|\mathbf{n}^{\mathrm{T}} \tilde{\mathbf{C}}_{\mathbf{l}}^{\prime \frac{1}{2}}\right\|^{2}=\sigma_{1}^{2} \cos ^{2} \theta+\sigma_{2}^{2} \sin ^{2} \theta$, we get

that is a $1 \mathrm{D}$ Gaussian.

$$
p\left(s \mid \theta, \mathbf{C}_{\mathbf{l}}^{\prime}\right)=\left|\frac{\mathrm{d} v}{\mathrm{~d} s}\right| \frac{1}{\sqrt{2 \pi}} \mathrm{e}^{-\frac{1}{2} v^{2}}=\frac{\mathrm{e}^{-\frac{1}{2} s^{2}\left(\sigma_{1}^{2} \cos ^{2} \theta+\sigma_{2}^{2} \sin ^{2} \theta\right)^{-1}}}{\sqrt{2 \pi\left(\sigma_{1}^{2} \cos ^{2} \theta+\sigma_{2}^{2} \sin ^{2} \theta\right)}}
$$

The lines parallel to $\mathbf{x}^{\prime \prime}$ intersect at $(\mathbf{d} 0)^{\mathrm{T}}$ in the transformed dual space that is equivalently the line that joins the origin $\mathbf{e}_{3}$ and $\mathbf{x}^{\prime \prime}$ in the transformed image space. In addition, we have parameterised the parallel lines in the transformed dual space by $s$ which represents the signed distance between the line $\mathbf{x}_{s}^{\prime \prime}$ and $\mathbf{e}_{3}$ in the transformed dual space. As we define $r$ as the signed distance from $\mathbf{e}_{3}$ in the transformed space, it also parameterises the points of line $(\mathbf{d} 0)^{\mathrm{T}}$ uniquely. Since the lines $\mathbf{x}_{s}^{\prime \prime}$ and points on the line $(\mathbf{d} 0)^{\mathrm{T}}$ have one-to-one correspondence, we have $s=s(r)$. In fact,

$$
s(r)=\left\{\begin{array}{ll}
1 / r, & \theta \in\left[-\frac{\pi}{2}, 0\right) \\
-1 / r, & \theta \in\left[0, \frac{\pi}{2}\right)
\end{array} .\right.
$$

Therefore the conditional probability density

$$
p\left(r \mid \theta, \mathbf{C}_{\mathbf{l}}\right)=\frac{1}{r^{2}} p\left(s(r) \mid \theta, \mathbf{C}_{\mathbf{l}}^{\prime}\right)=\frac{\mathrm{e}^{-\frac{1}{2} r^{-2}\left(\sigma_{1}^{2} \cos ^{2} \theta+\sigma_{2}^{2} \sin ^{2} \theta\right)^{-1}}}{r^{2} \sqrt{2 \pi\left(\sigma_{1}^{2} \cos ^{2} \theta+\sigma_{2}^{2} \sin ^{2} \theta\right)}} .
$$

Let us then consider the probability density $p\left(\theta \mid \mathbf{C}_{\mathbf{l}}\right)$ of the direction angle $\Theta=-\left(-\Theta^{\mathrm{c}}\right)^{\mathrm{c}}=$ $-\operatorname{arccot} L_{2} / L_{1}=-\arctan L_{1} / L_{2}$, where $\mathbf{L}=\left(L_{1} L_{2} L_{3}\right)^{\mathrm{T}} \sim N\left(\mathbf{e}_{3}, \mathbf{C}_{\mathbf{l}}^{\prime}\right)$. By substitution

$$
\mathbf{l} \triangleq\left(\begin{array}{l}
l_{1} \\
l_{2}
\end{array}\right)=\left(\begin{array}{c}
\sigma_{1} r^{\prime} \cos \theta^{\prime} \\
\sigma_{2} r^{\prime} \sin \theta^{\prime}
\end{array}\right)
$$


where $r^{\prime} \in \mathbb{R}, \theta^{\prime} \in\left[-\frac{\pi}{2}, \frac{\pi}{2}\right)$. Here, the determinant of the Jacobian is $\sigma_{1} \sigma_{2} r^{\prime}$, hence,

$$
p\left(r^{\prime}, \theta^{\prime} \mid \mathbf{C}_{\mathbf{l}}^{\prime}\right)=\sigma_{1} \sigma_{2}\left|r^{\prime}\right| p\left(\mathbf{l}\left(r^{\prime}, \theta^{\prime}\right) \mid \mathbf{e}_{3}, \mathbf{C}_{\mathbf{l}}^{\prime}\right)=\frac{\left|r^{\prime}\right|}{2 \pi} \mathrm{e}^{-\frac{1}{2} r^{\prime 2}} .
$$

The marginal probability over $r^{\prime}$ is

$$
p\left(\theta^{\prime} \mid \mathbf{C}_{\mathbf{l}}^{\prime}\right)=\int_{-\infty}^{\infty} p\left(r^{\prime}, \theta^{\prime} \mid \mathbf{C}_{\mathbf{I}}^{\prime}\right) \mathrm{d} r^{\prime}=\frac{1}{\pi} \int_{0}^{\infty} r^{\prime} \mathrm{e}^{-\frac{1}{2} r^{\prime 2}} \mathrm{~d} r^{\prime}=\frac{1}{\pi} .
$$

We may now transform $\theta^{\prime}$ back by the substitution $\theta^{\prime}=-\arctan \left(\frac{\sigma_{1}}{\sigma_{2}} \cot \theta\right)$, when the density function for $\theta$ is obtained as

$$
p\left(\theta \mid \mathbf{C}_{\mathbf{l}}\right)=p\left(\theta^{\prime} \mid \mathbf{C}_{\mathbf{l}}^{\prime}\right)\left|\frac{\mathrm{d} \theta^{\prime}}{\mathrm{d} \theta}\right|=\frac{\sigma_{1} \sigma_{2}}{\pi\left(\sigma_{1}^{2} \cos ^{2} \theta+\sigma_{2}^{2} \sin ^{2} \theta\right)} .
$$

The joint probability density $p\left(r, \theta \mid \mathbf{C}_{\mathbf{1}}\right)$ is now obtained by combining the results above,

$$
p\left(r, \theta \mid \mathbf{C}_{\mathbf{l}}\right)=p\left(r \mid \theta, \mathbf{C}_{\mathbf{l}}\right) p\left(\theta \mid \mathbf{C}_{\mathbf{l}}\right),
$$

and the claim follows.

Corollary 3 The probability density in (4), represented in coordinate basis of the second image, is

where

$$
p\left(x^{\prime}, y^{\prime} \mid \mathbf{C}_{\mathbf{l}}\right)=\left|\operatorname{det} \mathbf{J} \mathbf{r}\left(x^{\prime}, y^{\prime}\right)\right| p\left(\mathbf{r}\left(x^{\prime}, y^{\prime}\right) \mid \mathbf{C}_{\mathbf{l}}\right),
$$

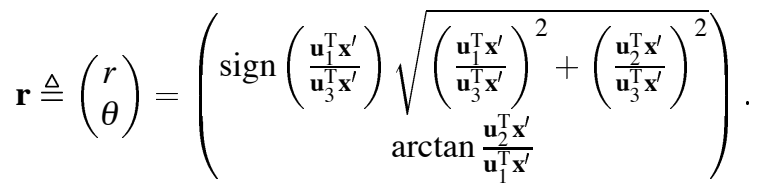

The derived probability density is nothing more than a transform of the dual, epipolar line density onto the image plane so it represents our degree of knowledge of the epipolar constraint: we do know that there is a true epipolar line but we do not know exactly where it is! The density however describes the probability, or certainty, that any point in the second view lies on the true epipolar line. Moreover, since the training data give evidence of the geometry only on certain disparities, the constraint will also be the most accurately known on similar disparities. Therefore the contours of the derived probability density are well-localised, bow-tie shaped and follow the shapes of the epipolar envelopes, as Fig. 4 illustrates. The narrowest location is around the point $\mathbf{u}_{2}$, and it is improbable that a match would reside near that point but deviated in the normal direction of the mean epipolar line. In addition, the contours quantify the risk of extrapolation beyond the training disparities on the mean epipolar line.

The probability density should be seen as the probabilistic equivalent for the estimated epipolar line. The density was derived under the Gaussian approximation for the epipolar line variation in the dual space which is, in fact, its exact asymptotic behaviour under certain general conditions. Moreover, when the amount of training data goes to infinity the epipolar geometry will generally become certain, and the probability density will converge to the true epipolar line. The density is neither ill-posed for nearly planar scenes but simply reports the evidence of the geometry that has been observed so far; likewise in Fig. 4(b), the density is also peaked around $\mathbf{u}_{2}$ strongly since the depth variation is relatively small in the scene, compare to Fig. 5 . In addition, since the probability density depends only on the uncertainty of the epipolar geometry, it can be directly used in a new scene (with the same camera configuration) to find new matches that can further refine the epipolar geometry by just showing new evidence of it! 


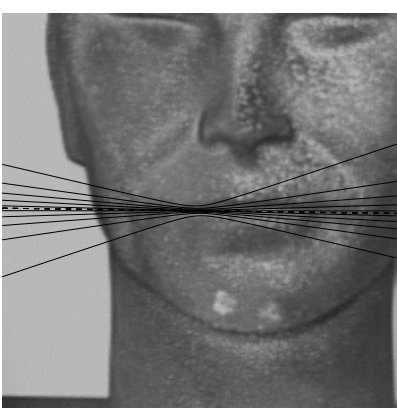

(a)

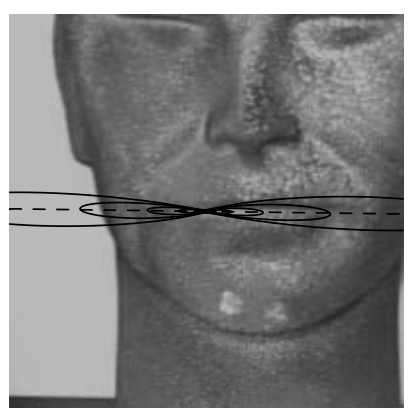

(b)

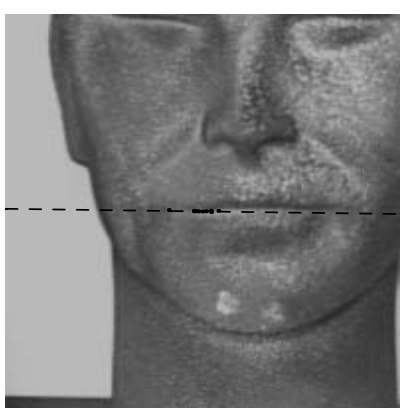

(c)

Figure 4: Comparison of epipolar envelopes and the epipolar line probability density, both derived from the covariance matrix of the fundamental matrix, as the point in the left corner of the mouth is given in the first image (not shown here). (a) Epipolar envelopes corresponding to $(1-k) \times 100 \%$ confidence intervals in [4], where $k=0.1,0.3,0.5,0.7,0.9$. (b) Equi-probability contours of the probability density (5) in the second image, at the levels of $10^{-5}, 10^{-4}, 10^{-3}, 10^{-2}$, and $10^{-1}$ times the maximum value at $\mathbf{u}_{2}$. (c) One thousand independent samples from the probability density (5). The estimated epipolar line is shown by the dashed lines; the robust estimation of the affine epipolar geometry and its uncertainty has been performed automatically from point correspondences as proposed in $[3,2]$. INRIA Syntim owns the copyright of the stereo pair.

\section{Conclusions}

In this paper, we have investigated the properties of the covariance matrix of the fundamental matrix. We first showed that the eigenvectors of the epipolar line covariance matrix have coupled point and line interpretations in the other image. In fact, given a point in one image, the most probable location in the other image is represented by the second eigenvector in the sense that it represents the pencil of epipolar lines with the largest total probability. Consequently, the previous observation of the narrowest point of the epipolar envelope was explained. The principal result of this paper is, however, the derivation of the probabilistic form of the epipolar constraint and we believe it will become an important tool for future applications since it is simply a rigorous representation of the degree of knowledge of the epipolar constraint on the image plane. The fundamental message is that we can not say more about the epipolar constraint than there is evidence of it in the training correspondences. In guided or dense matching applications, the derived probability density can be applied, for instance, to find new correspondences reliably whilst larger amount of training data may refine the epipolar geometry estimates and decrease the uncertainty. The scene reconstruction problem can be thus posed as a learning and inference problem, which starts from rough geometry estimates, and uses the scene itself to refine the geometry estimates and establish new correspondences in a systematic, iterative fashion. The derived density is additionally a most convenient prior in computing, for instance, a dense disparity map of a scene by Bayes methods.

\section{References}

[1] S. Brandt. Maximum likelihood robust regression with known and unknown residual models. In Proc. SMVP, in conjunction with ECCV 2002, pages 97-102, 2002.

[2] S. Brandt. Theorems and Algorithms for Multiple View Geometry with Applications to Electron Tomography. Doctoral thesis, Helsinki University of Technology, 2002. 


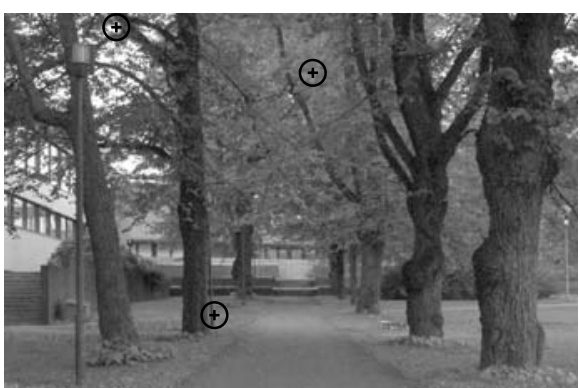

(a)

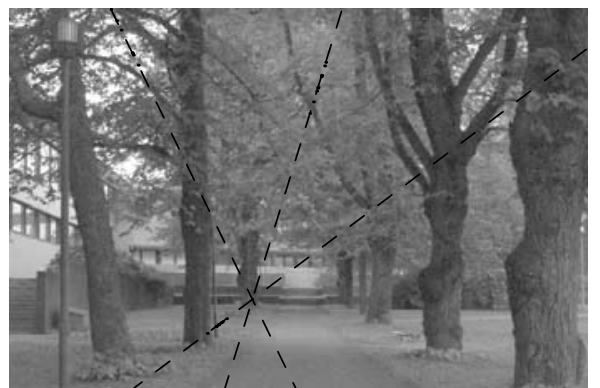

(b)

Figure 5: Behaviour of the epipolar line probability density when the epipolar geometry is known with a high level of accuracy. (a) Three randomly selected points in the first image. (b) One thousand independent samples, generated from each epipolar line probability density, lie in the vicinity of the mean epipolar line and show a large variation in disparity. The epipolar line corresponding to the point closest to the epipole in the first view is the most uncertain and the samples are more concentrated around the most probable point $\mathbf{u}_{2}$ on the line. The fundamental matrix and its covariance matrix were automatically robustly estimated from point correspondences as proposed in $[1,2]$.

[3] S. Brandt and J. Heikkonen. Optimal method for the affine F-matrix and its uncertainty estimation in the sense of both noise and outliers. In Proc. ICCV, volume 2, pages 166-173, 2001.

[4] G. Csurka, C. Zeller, Z. Zhang, and O. Faugeras. Characterizing the uncertainty of the fundamental matrix. CVIU, 68(1):18-36, 1997.

[5] O. Faugeras and Q.-T. Luong. Geometry of Multiple Images. MIT Press, 2001.

[6] R. Hartley and A. Zisserman. Multiple View Geometry in Computer Vision. Cambridge University Press, 2000.

[7] K. Kanatani. Optimal fundamental matrix computation: algorithm and reliability analysis. In Proc. Sensing via Imaging Information, pages 291-296, 2000.

[8] D. MacKay. Information Theory, Inference and Learning Algorithms. Cambridge University Press, 2003.

[9] P. Torr and A. Zisserman. Concerning Bayesian motion segmentation, model averaging, matching and the trifocal tensor. In Proc. ECCV, pages 511-527, 1998.

[10] B. Triggs. Joint feature distributions for image correspondence. In Proc. ICCV, volume II, pages 201-208, 2001.

[11] B. Triggs, P. McLauchlan, R. Hartley, and A. Fitzgibbon. Bundle adjustment a modern synthesis. In Vision Algorithms: Theory and Practice, volume 1883 of LNCS, pages 298-372, 2000.

[12] G. Xu and Z. Zhang. Epipolar Geometry in Stereo, Motion and Object Recognition. Kluwer Academic Publishers, 1996.

[13] Z. Zhang. Determining the epipolar geometry and its uncertainty: A review. IJCV, 27(2):161-195, 1998. 